\title{
COMPORTAMENTO DA PARASITEMIA AVALIADA PELO MÉTODO DE STROUT MODIFICADO EM CHAGÁSICOS AGUDOS EM TRATAMENTO
}

\author{
Cleudson Castro e Antonio Emanuel
}

\begin{abstract}
Em 18 pacientes com doença de Chagas aguda foi semiquantificada a parasitemia, pelo método de Strout modificado, antes e durante o tratamento. Antes da terapêutica a parasitemia variou entre 1 e 104 tripanossomos, e após o inicio do tratamento a parasitemia foi lida repetidamente com um intervalo, na maioria dos casos, entre dois e cinco dias, até a negativação. A dose inicial dos medicamentos foi de 10 a $15 \mathrm{mg} / \mathrm{kg} /$ dia de Nifurtimox para sete pacientes, e 10 a $20 \mathrm{mg} / \mathrm{kg} /$ dia de Benzonidazol para onze individuos. Após inicio do tratamento com Nifurtimox um paciente ficou o minimo de cinco e dois o máximo de 23 dias com parasitemia enquanto com o Benzonidazol um paciente permaneceu o máximo de 15 dias com parasitemia patente. $O$ Benzonidazol baixou a parasitemia mais rapidamente que o Nifurtimox.
\end{abstract}

Palavras-chaves: Doença de Chagas aguda. Parasitemia. Método de Strout modificado. Tratamento.

O método de Strout ${ }^{11}$, modificado por Flores e $\operatorname{cols}^{8}$ tem sensibilidade intermediária entre o xenodiagnóstico e o exame a fresco, para detectar a parasitemia na fase aguda da doença de Chagas ${ }^{3}$. No hospital de ensino da Universidade de Brasilia praticamente só é usado este método para diagnóstico e seguimento da fase aguda da moléstia 26 . Com a experiència acumulada, verificou-se quanto tempo o Trypanosoma cruzi permanece patente no sangue periférico de doentes agudos, tratados especificamente. Este será o objetivo do relato.

\section{CASUÍSTICA E MÉTODOS}

Entre 1980 e 1984 foram atendidos no hospital de ensino da Universidade de Brasilia, 35 pacientes com doença de Chagas aguda para tratamento especifico, a maioria proveniente do oeste da Bahia. Em 18 deles foi feito estudo semiquantitativo da parasitemia, antes e após inicio do tratamento até negativarem. Doze eram do sexo masculino, seis do sexo feminino com idade variável entre 1 e 65 anos, sendo 28,2 a média. A parasitemia de cada um foi semiquantificada repetidas vezes pelo método de Strout modificado por Emanuel e Castro ${ }^{6}$, com um intervalo, na maioria dos casos, entre dois e cinco dias. O metodo consiste em colher dez mililitros de sangue no adulto ou menos na criança, deixar à temperatura ambiente ou na estufa a

Trabalho realizado no Núcleo de Medicina Tropical e Nutrição e no Hospital Escola da Universidade de Brasilia Caixa Postal 153121, Brasília-DF, 70910 Brasil

Recebido para publicaçào em 12/5/88. $37^{\circ} \mathrm{C}$ durante uma a oito horas para retrair o coágulo. Transfere-se o sobrenadante para outro tubo e centrifuga a $19 \mathrm{G}$ por cinco minutos para sedimentar as hemácias. A seguir pipeta-se o soro para outro tubo, junto com poucas hemácias. Submete a centrifugação a 640 $\mathrm{G}$ durante dez minutos para concentrar os tripanossomos. Em seguida, mantendo a extremidade da pipeta próxima à superficie, aspira-se e despreza-se o soro, deixando-se apenas $0,5 \mathrm{ml}$ junto às hemácias, que são ressuspensas após agitar o tubo. Aspira-se 0,05 a 0,1 $\mathrm{ml}$ da suspensão, coloca-se em lâmina e cobre-se com laminula de 32 por 24 milimetros. Contam-se os tripanossomos em 50 campos de $400 \mathrm{x}$ assim distribuidos: dez em cada àngulo da laminula e dez no centro. Não encontrando parasitos, observam-se mais 300 campos. Deste modo foram feitos 26 exames antes e 91 após o início do tratamento. Em seis pacientes foram feitos mais de um exame antes do tratamento, porém, só foi registrado nas tabelas o que apresentou mais parasitos.

Os medicamentos usados no tratamento foram Nifurtimox e Benzonidazol. O primeiro foi ministrado em sete pacientes (tabela 1). Quatro iniciaram com a dose diária de $15 \mathrm{mg} / \mathrm{kg}$ e très com $10 \mathrm{mg} / \mathrm{kg}$, dividida em très tomadas, a cada oito horas, por um periodo que variou de 11 a 30 dias.

O Benzonidazol foi administrado em 11 pacientes (tabela 3). Cinco usaram inicialmente a dose diária de $15 \mathrm{mg} / \mathrm{kg}$, outros cinco tomaram $10 \mathrm{mg} / \mathrm{kg}$ e um usou $20 \mathrm{mg} / \mathrm{kg}$, distribuida em duas tomadas, a cada 12 horas por um periodo de 9 a 19 dias.

Em geral logo que a parasitemia negativou, a dose de ambos os medicamentos foi baixada para $7 \mathrm{mg} / \mathrm{kg} /$ dia; posteriormente a dose do Benzonidazol foi reduzida para $5 \mathrm{mg} / \mathrm{kg} / \mathrm{dia}$. Ambos os medicamentos foram mantidos por 60 dias. 


\section{RESULTADOS}

Na Tabela 1 estão os dados dos pacientes tratados com Nifurtimox. A quantidade de tripanossomos encontrados antes do tratamento variou de 3 a 45. Um paciente ficou o mínimo de 17 e outro o máximo de 46 dias desde o inicio da doença, sem tratamento especifico. O numero de exames positivos para cada paciente após inicio da terapeutica variou de um a sete, e o numero de dias que os pacientes permaneceram com parasitemia variou de 5 a 23 .

Os très primeiros pacientes permaneceram 17 a 23 dias após inicio do tratamento com parasitemia e a dose do medicamento foi diminuida antes que ela negativasse. Em geral, os indivíduos com parasitemias maiores antes do tratamento, tardaram mais a negativar os exames (casos um a quatro), o que contrasta com os casos cinco e sete, que tinham parasitemias menores. Entretanto, o caso seis faz exceção; tendo apenas très tripanossomos antes, permaneceu 23 dias com parasitemia após inicio da terapèutica (Tabelas 1 e 2). Os individuos que usaram $15 \mathrm{mg} / \mathrm{kg} /$ dia persistiram com parasitemia mais tempo em relação aos de $10 \mathrm{mg} / \mathrm{kg}$. Após o primeiro exame negativar os subse- qüentes ratificaram o resultado. Assim, très pacientes apresentaram très ou mais exames negativos, dois tiveram dois exames negativos, um apresentou um exame negativo e o caso quatro saiu de alta hospitalar com os exames positivos (Tabela 2 ).

Os dados daqueles que usaram Benzonidazol estão na Tabela 3 . O número de tripanossomos encontrados nos exames antes do tratamento variou e 1 a 104. Um paciente ficou o minimo de dez dias e outro o máximo de 42 dias, a partir do inicio da doença, sem tratamento especifico. O numero de exames positivos após o inicio do tratamento variou de um a quatro e um paciente permaneceu o máximo de 15 dias com parasitemia. Em todos, exceto no caso 16, a dose de medicação foi diminuida após a parasitemia negativar. A parasitemia baixou mais rapidamente nos que usaram $10 \mathrm{mg} / \mathrm{kg}$ em relação aos de $15 \mathrm{mg} / \mathrm{kg}$. Estes últimos ficaram entre 5 e 15 dias positivos, mas suas parasitemias antes do tratamento foram as mais altas. $O$ caso 16 não tinha parasitemia muito alta antes do tratamento, mas foi o que permaneceu positivo maior tempo. Os casos 8,13 e 14, após início do tratamento apresentaram os exames sempre negativos, mas suas parasitemias prévias foram as mais baixás.

Tabela 1 - Parasitemia em sete pacientes com doença de Chagas aguda tratados com Nifurtimox

\begin{tabular}{llccc}
\hline $\begin{array}{l}\text { No } \\
\text { e tempo inicial }\end{array}$ & $\begin{array}{c}\text { No de T. cruzi antes } \\
\text { do tratamento }\end{array}$ & $\begin{array}{c}\text { No de dias sem } \\
\text { tratamento após } \\
\text { iniciódoença }\end{array}$ & $\begin{array}{c}\text { No de exames }+ \\
\text { após inicio do } \\
\text { tratámento }\end{array}$ & $\begin{array}{c}\text { No de dias do útimo } \\
\text { exame }+ \text { após inicio } \\
\text { do tratamento }\end{array}$ \\
\hline
\end{tabular}

\begin{tabular}{llcccc}
\hline 1 & $15 \mathrm{mg} / \mathrm{kg}-14 \mathrm{~d}$ & ate $5 \mathrm{p} / \mathrm{c}$ & 39 & 3 & 17 \\
2 & $15 \mathrm{mg} / \mathrm{kg}-11 \mathrm{~d}$ & 35 & 17 & 7 & 22 \\
3 & $15 \mathrm{mg} / \mathrm{kg}-22 \mathrm{~d}$ & 45 & 18 & 6 & 23 \\
4 & $10 \mathrm{mg} / \mathrm{kg}-21 \mathrm{~d}$ & 17 & 43 & 2 & $11^{*}$ \\
5 & $10 \mathrm{mg} / \mathrm{kg}-15 \mathrm{~d}$ & 4 & 29 & 1 & 5 \\
6 & $15 \mathrm{mg} / \mathrm{kg}-30 \mathrm{~d}$ & 3 & 19 & 6 & 23 \\
7 & $10 \mathrm{mg} / \mathrm{kg}-24 \mathrm{~d}$ & 7 & 46 & 2 & $8^{* *}$
\end{tabular}

* não foram feitos exames posteriores, teve alta hospitalar

** usou Benzonidazol dois dias, antes de iniciar Nifurtimox

$\mathrm{p} c=$ por campo $\mathrm{d}=$ dias $t=$ positivo

Tabela 2 - Parasitemia em chagasicos agudos antes e durante o tratamento com Nifurtimox

\begin{tabular}{|c|c|c|c|c|c|c|c|c|c|c|c|c|c|c|c|c|c|c|c|c|c|c|c|}
\hline \multirow{2}{*}{\multicolumn{2}{|c|}{$\begin{array}{l}\text { No antes do } \\
\text { tratáninienito }\end{array}$}} & \multicolumn{22}{|c|}{ No de tripanossomos por dia, após início do tratamento } \\
\hline & & 2 & 34 & 5 & 6 & 7 & 8 & 10 & 1113 & 14 & 15 & 17 & 18 & 19 & 20 & 21 & 22 & 23 & 24 & 25 & 28 & 29 & 31 \\
\hline 1 & ate $5 \mathrm{p} / \mathrm{c}$ & & & & $1 / \mathrm{p} / \mathrm{c}$ & & 24 & & & & & 3 & & - & & - & & - & & & - & & \\
\hline 2 & 35 & & & & 4 & & & 1 & 8 & & 2 & & & & 2 & & 1 & & - & & - & & - \\
\hline 3 & 45 & & 13 & & & & 9 & & 4 & & 5 & & 2 & & & & & 1 & & - & & - & \\
\hline 4 & 17 & 4 & & & & & & & $2^{*}$ & & & & & & & & & & & & & & \\
\hline 5 & 4 & & & 2 & & & & - & & & & & & & & & & & & & & & \\
\hline 6 & 3 & 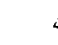 & 4 & & & 2 & & 3 & & 2 & & 1 & & & & & & 2 & & - & - & & - \\
\hline 7 & 7 & & & & & 3 & 1 & & & & & & & - & & & & & - & & & & \\
\hline
\end{tabular}

* não foram feitos exames posteriores, recebeu alta hospitalar

$\mathrm{p} / \mathrm{c}=$ por campo $\quad-=$ negativo 
Castro C, Emanuel A. Comportamento da parasitemia avaliada pelo método de Strout modificado em chagásicos agudos em tratamento. Revista da Sociedade Brasileira de Medicina Tropical 21: 177-180, Out-Dez, 1988

Tabela 3 - Parasitemia em onze pacientes com doença de Chagas aguda tratados com Benzonidazol

\begin{tabular}{rlcccc}
\hline No & $\begin{array}{l}\text { dose inicial } \\
\text { e tempo }\end{array}$ & $\begin{array}{c}\text { No de } T \text {. cruzi antes } \\
\text { do tratamento }\end{array}$ & $\begin{array}{c}\text { No de dias sem } \\
\text { tratamento apos } \\
\text { inicio doença }\end{array}$ & $\begin{array}{c}\text { No de exames }+ \\
\text { apó inicio do } \\
\text { tratamento }\end{array}$ & $\begin{array}{c}\text { No de dias do ultimo } \\
\text { exame + apos inicio } \\
\text { do tratamento }\end{array}$ \\
\hline 8 & $20 \mathrm{mg} / \mathrm{kg}-11 \mathrm{~d}$ & 1 & 21 & 0 & \\
9 & $15 \mathrm{mg} / \mathrm{kg}-9 \mathrm{~d}$ & 104 & 27 & 3 & 5 \\
10 & $10 \mathrm{mg} / \mathrm{kg}-9 \mathrm{~d}$ & 19 & 28 & 2 & 11 \\
11 & $15 \mathrm{mg} / \mathrm{kg}-19 \mathrm{~d}$ & 75 & 10 & 1 & 4 \\
12 & $10 \mathrm{mg} / \mathrm{kg}-12 \mathrm{~d}$ & 4 & 36 & 1 & \\
13 & $10 \mathrm{mg} / \mathrm{kg}-11 \mathrm{~d}$ & 4 & 15 & 0 & 8 \\
14 & $10 \mathrm{mg} / \mathrm{kg}-13 \mathrm{~d}$ & 2 & 42 & 0 & 15 \\
15 & $10 \mathrm{mg} / \mathrm{kg}-19 \mathrm{~d}$ & 55 & 19 & 2 & 5 \\
16 & $15 \mathrm{mg} / \mathrm{kg}-14 \mathrm{~d}$ & 27 & 34 & 4 & 10 \\
17 & $15 \mathrm{mg} / \mathrm{kg}-13 \mathrm{~d}$ & 80 & 27 & 2 & \\
18 & $15 \mathrm{mg} / \mathrm{kg}-11 \mathrm{~d}$ & 31 & 18 & 4 & \\
\hline
\end{tabular}

$\mathrm{d}=$ dias $\quad+=$ positivo

Tabela 4 - Parasitemia em chagásicos agudos antes e durante o tratamento com Benzonidazol

\begin{tabular}{|c|c|c|c|c|c|c|c|c|c|c|c|c|c|c|c|c|c|c|c|c|c|c|c|}
\hline \multirow[t]{2}{*}{ No } & \multirow{2}{*}{$\begin{array}{l}\text { antes do } \\
\text { tratamento }\end{array}$} & \multicolumn{22}{|c|}{ Ne de tripanossomos por dia, após inicio do tratamento } \\
\hline & & 1 & 2 & 3 & 4 & 5 & 6 & 7 & 8 & 9 & 10 & 11 & 12 & 13 & 14 & 15 & 16 & 17 & 18 & 19 & 21 & 28 & 33 \\
\hline 8 & 1 & & & & & & & - & & & & & & & & & & & & - & & & - \\
\hline 9 & 104 & 99 & & & & 7 & & & 3 & & & - & & - & & & & & & & & & \\
\hline 10 & 19 & & 1 & & & 1 & - & & & - & & - & & & & & & & & & & & \\
\hline 11 & 75 & & & & & & & & & & & 14 & & & & & & - & & & & - & \\
\hline 12 & 4 & & & & 2 & & & & - & & - & & & - & & & & & & & & & \\
\hline 13 & 4 & & & & & - & & & & & - & & - & & & & & & & & & & \\
\hline 14 & 2 & & & & & - & & & & & & & - & & & & & & & & & & \\
\hline 15 & 55 & & & 26 & & & & & 1 & & - & & - & & - & & & & & & & & \\
\hline 16 & 27 & & 5 & & & & & 1 & & & & 2 & & & & 1 & - & & - & & - & & \\
\hline 17 & 80 & & & 1 & & I & & - & & & - & & - & & & & & & & & & & \\
\hline 18 & 31 & & 15 & & 10 & & & 8 & & & 11 & & & & - & & - & & & & & & \\
\hline
\end{tabular}

Após o último exame positivo, oito pacientes tiveram très exames negativos e très apresentaram dois exames negativos pelo método de Strout (Tabela 4). Em quatro pacientes que usaram Nifurtimox e quatro que tomaram Benzonidazol, no primeiro dia em que a pesquisa de tripanossomos foi negativa nos 50 campos, o exame de mais 300 campos mostrou um ou dois parasitos, sendo os exames subseqüentes negativos.

\section{COMENTÁRIOS}

A evoluçāo da parasitemia patente de chagásicos agudos em tratamento é variavel, podendo desaparecer em poucos dias ${ }^{7}$ ou persistir por mais de 30 dias $^{9}$ e fatores como, tipo de cepa, medicamento utilizado e o metodo parasitologico usado devem influenciar. Nessa experiència o desaparecimento da parasitemia após o início do tratamento deu-se precocemente em alguns pacientes e tardiamente em outros.
Esse fato deve ter importancia pratica na orientaçāo do tratamento, ponderando que a medicaçào na fase aguda e iniciada com dose elevada, para em seguida ser diminuida, devido a toxidade. Em geral a redução da dosagem é baseada na melhora clínica, porém, se for feito o exame parasitologico, é possível ainda detectar parasitemia patente. Esse fato ocorreu com os tres primeiros pacientes medicados com Nifurtimox.

A evolução da parasitemia de chagasicos agudos em tratamento pode ser avaliada examinando o sangue a fresco ${ }^{1}$. Entretanto, esse metodo diagnostica somente $34 \%$ dos casos agudos, enquanto o Strout diagnostica $70 \%$ e o xenodiagnostico $97 \% 3$. Este ultimo apesar de ser o metodo mais sensivel e estabelecer o diagnóstico 5 a 25 dias apos o repasto sangüineo 410 fornece resultado mais tardio que o Strout e é feito quase exclusivamente nas instituições de pesquisa e universitarias. O metodo de Strout é simples, exeqüivel em qualquer laboratório e fornece 
resultado imediato. Esse método aplicado a cada três a cinco dias pode, juntamente com a clínica, orientar sobre o momento apropriado em que a dose do medicamento deve ser reduzida.

Para ambos os medicamentos, a dose inicial de $10 \mathrm{mg} / \mathrm{kg} /$ dia baixou a parasitemia mais rápido que $15 \mathrm{mg} / \mathrm{kg}$. A análise das tabelas 2 e 4 destaca que o Benzonidazol baixou a parasitemia mais rapido que o Nifurtimox. A média geométrica do maior tempo que cada paciente permaneceu com parasitemia, foi 4,3 dias para o Benzonidazol e 13,6 para Nifurtimox. Com o último, dois pacientes permaneceram 23 dias com parasitemia, enquanto com o Benzonidazol um paciente passou o máximo de 15 dias.

\section{SUMMARY}

In 18 patients with acute Chagas' disease parasitaemia was semi quantitated before and during treatment using a modified Strout method. Before therapy the parasitaemia varied between 1 and 104 trypanosomes and after begining treatment the parasitaemia was estimated repeatedly every two to five days until it fell below detectable levels. The initial dose of drug was 10 to $15 \mathrm{mg}$ per kilogram per day of Nifurtimox for seven patients and 10 to $20 \mathrm{mg}$ per kilogram per day of Benzonidazol for eleven patients. After start of treatment with Nifurtimox the duration of patient's parasitaemia varied from 5 to 23 days. With Benzonidazole one patient had parasitaemia for 15 days. Benzonidazole depressed parasitaemia more rapidly than Nifurtimox.

Key-words: Acute Chagas disease. Parasitaemia. Strout method modificated. Treatment.

\section{REFERÊNCIAS BIBLIOGRÁFICAS}

1. Cançado JR, Salgado AA. Efeito do Benzonidazol sobre a parasitemia da doença de Chagas aguda humana. In: Resumos da IX reunião anual sobre pesquisa básica em doença de Chagas, Caxambu, p. 144, 1982.

2. Castro C, Emanuel A. Evolução da parasitemia de chagásicos agudos em tratamento, avaliada pelo método de Strout. In: Resumos do XIX Congresso da Sociedade Brasileira de Medicina Tropical, Rio de Janeiro, p. 30, 1983.

3. Cedillos RA, Dimas D, Hernandez AY. Blood concentration method in the diagnosis of Chagas' disease. Revista Latino-Americana de Microbiologia 12: 201-203, 1970.

4. Dias JCP. Doença de Chagas em Bambui, Minas Gerais, Brasil. Estudo clinico epidemiologico a partir da fase aguda, entre 1940 a 1982 . Tese de Doutourado. Universidade Federal de Minas Gerais, Belo Horizonte, 1982.

5. Dias JCP, Dias E. Consideraçōes gerais acerca de 67 casos humanos de doença de Chagas aguda, observados pelo Posto Dr. Emmanuel Dias (Instituto Oswaldo Cruz), Bambui, MG, entre 1955 e 1967. O Hospital 73: 1935-1945, 1968.

6. Emanuel A, Castro CN. Método de Strout utilizando diferentes velocidades de centrifugação no diagnóstico da fase aguda da doença de Chagas. Revista da Sociedade Brasileira de Medicina Tropical 18: 247-249, 1985.

7. Fernandez JJ, Cedillos RA, Godoy GA. Tratamiento dz la enfermedad de Chagas aguda con Bay 2502. Boletin Chileno de Parasitologia 24: 51-53, 1969.

8. Flores MA, Trejos A, Paredes AR, Ramos AY. El método de concentración de Strout en el diagnóstico de la fase aguda de la enfermedad de Chagas. Boletin Chileno de Parasitologia 21: 38-39, 1966.

9. Rebosolan JB. Sensibilidad de los métodos de diagnostico parasitologico en pacientes con enfermedad de Chagas aguda tratados con Bay 2502. Boletin Chileno de Parasitologia 24: 49-50, 1969.

10. Rohwedder RW, del Prado CE, Cerisola JA, Rebosolan JB. Aportes al metodo del examen del xenodiagnóstico previo licuado de los triatominos. Boletin Chileno de Parasitologia 25: 106-110, 1970.

11. Strout RG. A method for concentrating hemoflagellates. The Journal of Parasitology 48: 100, 1962. 